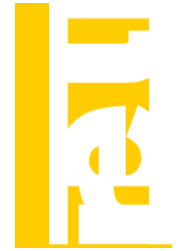

\title{
LA MEDICIÓN DEL EMPLEO Y EL PARO EN LA OBRA DE LUIS TOHARIA
}

\author{
Miguel Á. Malo*, José Ignacio Pérez Infante ${ }^{\dagger}$ \\ * OIT y Universidad de Salamanca \\ $\dagger$ Economistas Frente a la Crisis
}

\section{Resumen}

En este artículo se revisan los trabajos que desde la década de los ochenta realizó Luis Toharia sobre la medición del empleo y de paro en España. La revisión se centra tanto en las encuestas (sobre todo la Encuesta de Población Activa) como en las fuentes administrativas (el Paro Registrado en el caso del desempleo).

Palabras clave: Desempleo, empleo, Encuesta de Población Activa, Paro Registrado.

Clasificación JEL: J64, C81.

\begin{abstract}
In this article, the authors present a survey of the different articles published by Luis Toharia from the 80's about employment and unemployment measurement in Spain. This review focuses in measures coming from households surveys (mainly the Labour Force Survey) and administrative sources (as the records of the Registered Unemployment)

Key words: Unemployment, employment, Labour Force Survey, Registered Unemployment. JEL Classification: J64, C81.
\end{abstract}

\section{Introducción}

Un elemento distintivo y muy destacado de la investigación de Luis Toharia es la constante preocupación por la calidad de la información y de los datos utilizados en el análisis del mercado de trabajo. Esta preocupación se ha plasmado de forma muy clara en el estudio de las fuentes de los datos de empleo y paro en España, en particular de la Encuesta de Población Activa y el Paro Registrado.

Además, sus resultados han influido de forma clave (y fructífera) sobre cambios metodológicos de dichas fuentes (Jimeno y Pérez Infante, 2012). En este sentido, destaca la relevante participación de Luis Toharia en el Grupo de Trabajo sobre Estadísticas Coyunturales del Mercado de Trabajo del Consejo Superior de Estadística, desde su creación en 1998, que ha influido de forma crucial en los cambios metodológicos que ha ido

(C) Revista de Economía Laboral 
produciéndose en la EPA, desde entonces, principalmente los de 1999, 2002 y 2005. También en el ámbito de las mejoras de las estadísticas relativas al mercado de trabajo ha sido decisiva su participación en los estudios sobre los problemas y limitaciones del paro registrado que llevó a concienciar a las autoridades políticas del Ministerio de Trabajo sobre la necesidad de modificar el propio sistema del paro registrado, lo que provocó la puesta en marcha del Sistema de Información de los Servicios Públicos de Empleo (SISPE) en 2005, en cuyo diseño y desarrollo su asesoramiento y participación fueron cruciales.

También en el seno del citado Grupo de Trabajo de Estadísticas Coyunturales del Mercado de Trabajo intervino muy activamente en la elaboración para distintos años de las armonizaciones y comparaciones entre los datos de empleo de la EPA y de las afiliaciones en situación de alta laboral de la Seguridad Social y entre los datos del paro de la EPA y del paro registrado en las oficinas públicas de empleo.

Asimismo, Luis Toharia fue pionero en el análisis de los microdatos de empleo y el paro de la EPA y de los Servicios Públicos de Empleo, así como de las cifras obtenidas de la Muestra Continua de Vida Laboral (MCVL) de la Seguridad Social y el enlace de los datos de esta muestra con los de otras estadísticas de los Servicios Públicos de Empleo (como la de Contratos Registrados).

Las aportaciones de Luis Toharia a la medición del empleo y del paro es el objetivo de este artículo, que se divide en dos apartados. El primero está dedicado a los distintos trabajos analíticos publicados por Luis Toharia sobre la medición del empleo y el paro de la EPA. El segundo se centra en los trabajos realizados sobre la estadística del paro registrado, publicada por el Servicio Público de Empleo Estatal (antiguo INEM), algunos de los cuáles sirvió, como se ha señalado, de base para el cambio en el diseño y metodología del paro registrado en 2005 y la creación y desarrollo del SISPE. El artículo finaliza con un apartado de conclusiones sobre la importancia de las aportaciones de Luis Toharia en materia de estadísticas del mercado de trabajo.

\section{La medición del empleo y el paro en la EPA}

Son muy abundantes los artículos y trabajos de investigación de Luis Toharia relativos a la EPA, estadística que siempre defendió como la más completa y fiable para la medición de las distintas situaciones de las personas en relación con la actividad económica y, más concretamente, con el mercado de trabajo (actividad e inactividad, empleo y paro), incluso en los momentos de mayor incomprensión de esta estadística y de críticas a la 
misma (especialmente cuando aumentaba el paro estimado por la EPA) por parte principalmente de miembros de los distintos gobiernos (sobre todo de los ministros y altos cargos de los ministerios de Economía y de Trabajo), pero también por políticos de distintos partidos políticos y representantes del mundo empresarial.

En estos trabajos Luis Toharia ha considerado y analizado, siempre con un espíritu crítico pero constructivo, prácticamente todos los temas relacionados con la EPA, como las definiciones, las variables estimadas, la metodología, el contenido, el proceso de elaboración de la encuesta y de sus resultados, la relación con otras estadísticas, como la Contabilidad Nacional de España, el paro registrado y las afiliaciones de la Seguridad Social, la calidad y fiabilidad de las estimaciones de la encuesta, sus cambios metodológicos, las rupturas de la homoneidad de las series históricas que supusieron la mayoría de esos cambios metodológicos, etc.

En este artículo por razones relacionadas principalmente con las limitaciones de espacio sólo se van a considerar las aportaciones de Luis Toharia a unas pocas de esas materias, a aquellas que desde la perspectiva actual y la situación presente del mercado de trabajo parecen más transcendentales y sus aportaciones son más relevantes, sin que ello signifique que las otras materias estudiadas por Luis Toharia en relación con la EPA no sean importantes.

Estas materias van a ser tres. En primer lugar el significado y contenido de las definiciones de la EPA del empleo y el paro y la relación cuantitativa existente entre esas dos variables, lo que explicaría la superioridad de la EPA sobre otras estadísticas relacionadas con el mercado de trabajo, así como la crítica a la utilización errónea y deficiente en muchas ocasiones, algo que desgraciadamente persiste en la actualidad, de la relación y significado de esas dos variables por parte, especialmente, de muchos políticos. En segundo lugar, y, en parte, relacionada con lo anterior, la posibilidad de que las estimaciones del empleo de la EPA estén infravaloradas, lo que puede ser cierto, sobre todo hasta los cambios metodológicos de la EPA de 2002, y las justificaciones sobre que esas posibles infraestimaciones del empleo de la encuesta no implican necesariamente la sobrevaloración de las estimaciones del paro de la EPA. En tercer lugar, se presentan los elementos de la discusión sobre si hay una relación entre el paro y la economía subterránea. Y, en cuarto lugar, el análisis específico del cambio de definición del paro de la EPA en 2002 en relación con los llamados "buscadores pasivos de empleo"; los que únicamente buscan empleo registrándose como demandante de empleo en una oficina pública de empleo. 


\subsection{En torno a las definiciones}

Dos artículos fundamentales para el análisis de las definiciones y los problemas estadísticos que plantean las dos situaciones relacionadas con la actividad de las personas en el mercado de trabajo, el empleo y el paro, son el publicado en 1996 en Cuadernos de Información Económica ${ }^{\circ}$ 108, "La medición del empleo y el paro en España"1, y el publicado en el número 0 de la Revista Gallega de Empleo, "El paro en España: ¿puede ser tan alto?"2.

En ambos Luis Toharia se plantea las divergencias existentes en la mediación del empleo entre las estimaciones de la EPA y de las afiliaciones en situación de alta laboral de la Seguridad Social y en la medición del paro entre las estimaciones de la EPA y el paro registrado, es decir, entre los resultados de la encuesta y dos estadísticas administrativas.

En esos dos artículos y en algunos trabajos más, como en el apartado correspondiente al análisis de la EPA del libro sobre el mercado de trabajo que dirigió Luis Toharia en $1998^{3}$ y en un artículo muy anterior publicado en Debats en $1988^{4}$, Luis Toharia analiza las definiciones de empleo y paro de la EPA y compara la medición de esas variables estimadas por la encuesta con la de las estadísticas administrativas citadas, destacando dos cuestiones importantes desde el punto de vista estadístico: la primera, que la EPA es la única estadística que mide, simultáneamente, las dos situaciones de empleo y paro y, la segunda, que la EPA sigue los criterios estrictos de medición de ambas variables de la OIT y de Eurostat, algo que no es el caso de las dos estadísticas administrativas, y que convierte a la encuesta en una estadística superior a las otras dos y perfectamente comparable con las otras encuestas sobre la fuerza de trabajo del resto de los países europeos y de la OCDE.

En lo que respecta a las definiciones de empleo y paro de la EPA, hace mención a una diferencia relevante entre las dos magnitudes: ya que, si en el caso de la situación de empleo, definida como aquella en la que la persona ha trabajado en la semana de referencia (la anterior a la de la entrevista), al menos, una hora, el concepto es de naturaleza claramente objetiva, máxime, si se tiene en cuenta, que la persona encuestada no se autoclasifica como ocupada o no, sino que la clasificación en relación con el

1 Toharia (1996a). Este artículo se incluye en el libro del Ministerio de Empleo y Seguridad Social de homenaje a Luis Toharia (Jimeno y Pérez Infante, 2012; págs. 205279).

2 Toharia (2000)

3 Toharia (1998)

${ }^{4}$ Toharia (1988), reproducido en Bentolila y Toharia (1991; págs. 75-88). 
empleo es el resultado de las contestaciones a una serie de preguntas, relacionadas con si tiene o no empleo y si ha trabajado o no, al menos, una hora; en el caso de la situación del paro, la definición es más compleja y difícilmente puede abstraerse de la subjetividad de la persona entrevistada.

En efecto, Luis Toharia al insistir en que la persona parada es aquella persona en edad de trabajar que no tiene empleo en la semana de referencia (no ha trabajado ni una hora), pero que, además, tiene que cumplir otras dos condiciones no tan objetivas, como estar buscando activamente empleo y estar disponible para trabajar, destaca la complejidad de la medición de esta variable, complejidad muy superior a la del empleo.

Pero la complejidad de la medición del paro por la EPA, en ningún momento, insiste Luis Toharia, debería cuestionar su superioridad sobre el paro registrado en las oficinas públicas de empleo. La razón básica es que esta última estadística (de carácter administrativo) sólo considera a los demandantes de empleo que utilizan como método de búsqueda la inscripción en las oficinas públicas de empleo, excluyendo a los que utilizan otros métodos distintos de búsqueda de empleo y no se inscriben en esas oficinas, y, además, excluye a una serie de demandantes de empleo no ocupados inscritos en las oficinas, con base en los criterios establecidos en una Orden Ministerial de 1985, que, pese a esa exclusión, podrían cumplir las condiciones de búsqueda y disponibilidad de la OIT para ser considerados como parados y que en el supuesto de cumplir esas condiciones se considerarían como tales en el caso de la Encuesta, lo que en palabras de Luis Toharia "Ilevaría a descartar el uso del paro registrado en favor del de la EPA"

Sin embargo, según Luis Toharia, la utilización que suele hacerse por muchas personas, especialmente periodistas y políticos, de estos conceptos de empleo y paro puede llegar a ser muy incorrecta, cuando no distorsionante.

En este sentido, destaca lo que en Toharia (1996a) se califica como la "cuadratura del círculo" entre el empleo y el paro, y que consiste en considerar que toda variación del paro supone una variación de la misma cuantía pero de distinto sentido del empleo y viceversa, sin tener en cuenta la variación de la población activa; ya que puede ocurrir, por ejemplo, que un aumento del empleo no se corresponda con un descenso del paro de la misma cuantía sino que, al menos, en parte, se deba a un aumento de la población activa procedente de personas hasta entonces inactivas. Esta confusión es algo que desgraciadamente se mantiene

5 Toharia (1998; pág. 48). 
actualmente, a pesar de las constantes denuncias que sobre esta cuestión hizo Luis Toharia.

\subsection{El paro registrado y las críticas a la EPA}

Luis Toharia se opuso sistemáticamente a las críticas que muchos políticos, incluso con responsabilidades gubernamentales, hicieron a la fiabilidad de la EPA sobre todo en épocas en las que aumentaba persistente e intensamente la cifra del paro y en las que el paro estimado por la EPA superaba con gran diferencia al paro registrado en las oficinas públicas de empleo.

En Toharia (1996a y 1998) y más tardíamente en Toharia (2000), se señala que, pese al reconocimiento general por parte de los expertos y analistas de la corrección teórica de la estimación del paro por la EPA, su cifra se ha visto envuelta en dos oleadas de críticas que han coincidido con dos momentos de crisis padecidas por la economía española, 1985 y $1994^{6}$.

La oleada de críticas de 1985 coincidió con la superación de la tasa de paro por primera vez de la barrera del $20 \%$, debido a la creencia de muchos políticos de entonces de la existencia de una elevada proporción de economía sumergida, y la de 1994, aunque menos específica, iba dirigida, según Luis Toharia, directamente al corazón de la encuesta, al considerar personas relevantes del gobierno, como el entonces ministro de trabajo, José Antonio Griñan, que era más fiable para la medición del paro el registro en las oficinas públicas de empleo que el estimado por la EPA. Desgraciadamente, las críticas políticas a la EPA, aunque posiblemente no con tanta rotundidad como en 1994, no se han abandonado en la actual situación de recesión económica, en la que la tasa de paro ha superado el $25 \%$ en el tercer trimestre de 2012.

Pero, a pesar de la corrección general de los datos de la EPA y, en particular de sus definiciones, uno de los aspectos que analiza Luis Toharia con más detalle en distintos artículos ${ }^{7}$ es la posible infravaloración de la estimación del empleo de la EPA, especialmente si se compara con el dato de afiliaciones a la Seguridad Social y si se tiene en cuenta que esta última estadística no considera a los trabajadores ocupados irregularmente y la EPA, en principio, y, al menos en parte, si que los considera. Esta infravaloración del empleo de la EPA no se debería, según Luis Toharia, a los problemas relacionados con la definición del empleo de la encuesta, que, además, al ser tan amplia como que basta

\footnotetext{
${ }^{6}$ Toharia (1998; págs. 44 y 45).

${ }^{7}$ En los citados artículos Toharia (1996a; 1996 b; 1998; y 2000) y en algunos otros como Toharia (2001) y Garrido, Requena y Toharia (2000).
} 
con trabajar sólo una hora en la semana de referencia para que se compute a una persona como ocupada, pareciera que, incluso, podría llegar a sobrevalorar las cifras reales de empleo. Por lo tanto, la infravaloración del empleo debe deberse a otras razones, en particular, según Luis Toharia, sobre todo, a los problemas relacionados con el proceso de elaboración de la encuesta.

En este sentido, la razón principal de la infravaloración del empleo de la EPA sería, según Luis Toharia, la tendencia al envejecimiento de la muestra de la encuesta, a que la muestra va paulatinamente perdiendo representatividad de la población real, sobre todo en lo que respecta a la estructura por edades de esa población.

Para entender este problema Luis Toharia se basa en el procedimiento de muestreo elegido por el INE para seleccionar la muestra y en el proceso de renovación trimestral de esa muestra. En efecto, el procedimiento de selección de la muestra es bietápico, de forma que en la primera etapa se seleccionan las secciones censales con base en los resultados de un Censo de Población anterior, de hace años, y en la segunda etapa se eligen aleatoriamente un número de hogares familiares dentro de cada sección censal. Y, por su parte, la renovación de la muestra, a razón de una sexta parte de los hogares cada trimestre, se venía produciendo manteniendo fijas, sin renovar, las secciones censales. Este procedimiento de renovación de la muestra, según Luis Toharia, explicaría un desfase de la muestra en relación con la población real en cada momento y, en concreto, una distribución por edades de la muestra muy diferente de la distribución de la población real, infravalorándose en la muestra la población de 25 a 50 años, la potencialmente más activa, y sobrevalorándose la población menor de 25 años y la mayor de 55 años, con menores tasas de actividad que la media; es decir, el proceso de elaboración de la encuesta estaría infravalorando la población activa y, por lo tanto, la población ocupada.

Pero a esta razón, de infravaloración del empleo, por el envejecimiento de la muestra o desactualización del marco de la población de referencia de la muestra, Luis Toharia añade otra, relacionada con el trabajo de campo, con las dificultades de la encuesta para encontrar a las personas ocupadas o, lo que es lo mismo, las dificultades de la encuesta para realizarla a determinados hogares, en los que todas las personas activas están ocupadas ${ }^{8}$, lo que se agrava con el tratamiento de esos hogares en la encuesta que, si siguen sin ser entrevistados sus

8 Toharia (1998; pág. 54). 
componentes, acabarán suprimiéndose de la muestra sin ser sustituidos por otros hogares ${ }^{9}$.

En Toharia (1996a; 1998 y 2000) se analiza con detalle el efecto de la renovación que el INE realizó de las secciones censales de la encuesta en su totalidad, entre el tercer trimestre de 1995 y el segundo trimestre de 1996, a razón de una sexta parte en cada una de los seis trimestres del período, para adaptar la muestra a la población (nivel y estructura por edades) del Censo de 1991. Esta revisión del marco poblacional de la encuesta (secciones censales) corrigió, en gran medida, el envejecimiento de la muestra sobre todo, la infravaloración de la población activa y de la ocupada, aunque, al adaptarse, al final de la revisión de la muestra, el segundo trimestre de 1996, a una población de cinco años antes y al no superarse el otro problema, el de la dificultad de encontrar ocupados a través de las entrevistas, la infravaloración de esas dos poblaciones, la activa y la ocupada, no se superó en su totalidad.

Ahora bien, la insistencia de Luis Toharia en estos problemas de la EPA y su activa participación en el citado Grupo de Estadísticas Coyunturales del Mercado de Trabajo del Consejo Superior de Estadística ayudó a la puesta en marcha de otras reformas del diseño y metodología de la EPA introducidas por el INE, como las de 1999, 2000, 2002 y 2005, que volvieron a adaptar y rediseñar las secciones censales de la muestra, actualizaron, las de 2002 y 2005 , las proyecciones demográficas base de la encuesta, introdujeron modificaciones en los coeficientes de elevación de la muestra a la población de referencia, como la de 2002, que reponderó la estructura por edades de la muestra, y a partir de 2005 se actualizaron anualmente las secciones censales al Padrón Continuo de Población, lo que paulatinamente ha podido ir superando esas limitaciones y problemas de infravaloración de la población activa y el empleo.

En Toharia (2001), mediante la reconstrucción de una serie homogénea de la EPA, se muestra que las reformas introducidas en la EPA en el período 1995-96 y en el año 1999 produjeron un afloramiento estadístico del empleo y no así del paro, de forma que las tasas de variación interanuales de la serie reconstruida del empleo eran muy superiores a las de la serie publicada originalmente por el INE, lo que era sintomático de la corrección, al menos parcial, de la señalada infravaloración del empleo por parte de la EPA. Al mismo tiempo, es una prueba indirecta de que los problemas tantas veces debatidos de la EPA afectaban sobre todo a la medición del volumen de empleo y no del paro, como Luis Toharia había sostenido desde un primer momento.

${ }_{9}^{9}$ Pérez Infante (2006; págs. 73-75). 
Ahora bien, en contra de lo que señalaban muchas de las críticas de la EPA, Luis Toharia insistió mucho y defendió con enorme vigor que la posible infravaloración del empleo no provocaba necesariamente la sobrevaloración del paro, ya que la infravaloración del empleo procedía más de la sobrevaloración de la población inactiva que de la sobrevaloración del paro. Es decir, que personas realmente ocupadas y no consideradas como tales por las razones anteriormente citadas por la EPA eran, en su mayoría, computadas como inactivas, pero no como paradas. ${ }^{10}$

\subsection{El paro y la economía sumergida}

Toharia (1998) y Toharia (2000) ejemplifican la manera clara y drecta en que Luis Toharia trató el problema de la relación entre el paro y la economía sumergida. Esta línea de trabajo arrancó ya muy atrás, con el análisis de los datos de la Encuesta de Condiciones de Vida y Trabajo (ECVT) de 1985, promovida por el Gobierno de entonces con la finalidad política de demostrar que el paro estimado por la EPA estaba enormemente sobreestimado, en gran parte debido a la economía sumergida $^{11}$. En la ECVT esta incidencia de la economía sumergida se relacionaba con el concepto de empleo irregular de los trabajadores respecto a la Seguridad Social. Los resultados de la ECVT no fueron concluyentes y Luis Toharia señaló que la nueva encuesta no refutaba los datos de la EPA, sino que confirmaba que esa encuesta era capaz de captar una parte significativa de las personas que trabajaban en condiciones irregulares, y que se podrían considerar que formaban parte de la economía sumergida u oculta ${ }^{12}$.

10 En Toharia (1996a) se exponen de forma sistemática tres comparaciones con otras fuentes por las que la hipótesis de la sobrevaloración del paro estimado por la EPA no es consistente: la comparación con el paro medido a través del Censo de Población; la medición del paro por autoclasificación en la Encuesta Demográfica de la Comunidad de Madrid; y la autoclasificación como parado y las preguntas sobre búsqueda de empleo de la Encuesta Socio-Demográfica (realizada por el INE en 1991 y con un tamaño muestral tres veces superior al de la EPA).

${ }^{11}$ En Muro, Raymond, Toharia y Uriel (1989) se analizan los aspectos relacionados con el diseño de la ECVT en comparación con la EPA, así como la clasificación de la población y la comparación de los resultados de las dos encuestas. No hay que confundir esta encuesta realizada una sola vez en 1985 con la Encuesta de Condiciones de Vida en el Trabajo, que realiza anualmente el Ministerio de Empleo y que los investigadores laborales españoles también suelen denominar ECVT.

12 Además de en Toharia (1998 y 2000), el tema de la relación entre el paro y la economía subterránea se trata en otros artículos, más concretamente en la participación de Luis Toharia en el V Informe FOESSA (Toharia, 1994), con base en la encuesta el FOESSA relativa a 1993, y en el citado artículo de Ekonomiaz, Toharia (1996b). 
Adicionalmente a la problemática de la economía subterránea y, en parte, relacionada con esa problemática, una de las razones que suele utilizarse por los críticos de la EPA para justificar la sobrevaloración de las estimaciones del paro de la encuesta es la ocultación en la misma por parte de algunas personas beneficiarias de las prestaciones por desempleo de que están ocupadas. Pero Luis Toharia advirtió muy pronto de que al clasificarse a las personas encuestadas en las distintas situaciones relacionadas con la actividad económica de acuerdo con unos criterios objetivos, después de contestar a una serie de preguntas en las que no se recoge explícitamente si se encuentran desempleados o no, la ocultación de la situación de la persona entrevistada ni es fácil ni puede ser muy significativa, o, lo que es lo mismo, llegar a tener un carácter muy generalizado.

Ahora bien, desde 1987 la EPA pregunta expresamente a las personas entrevistadas si cobran o no prestaciones por desempleo y de las contestaciones a esta pregunta se deduce que, aunque las cifras de la EPA y las de los beneficiarios de las prestaciones por desempleo obtenidas del Servicio Público de Empleo Estatal tienen una tendencia similar, las primeras son algo inferiores a las segundas, lo que significa que en la EPA hay perceptores de prestaciones por desempleo que no se identifican como tales, pero que ello no supone necesariamente que toda esa diferencia de personas represente una sobrevaloración del paro, máxime cuando la pregunta de la situación respecto a la actividad estaba al principio del cuestionario y las relacionadas con las oficinas públicas de empleo al final del mismo ${ }^{13}$.

\subsection{La intensidad de la búsqueda de empleo}

Otra de las cuestiones relacionadas con la EPA que analizó con detalle Luis Toharia fue el de la intensidad de la búsqueda de empleo por parte de los parados ${ }^{14}$. La búsqueda poco intensa de un número elevado de personas paradas haría que en realidad esas personas fueran externas al mercado de trabajo y no presionaran a la baja sobre los salarios. Luis Toharia insistió mucho en que la intensidad de la búsqueda era muy difícil de medir y que esa intensidad puede depender de los métodos de búsqueda utilizados por los entrevistados. En concreto, ese podía ser el caso del colectivo de personas paradas cuyo único método de búsqueda declarado en la encuesta es la inscripción como demandante de empleo en una oficina pública de empleo, que, aunque con tendencia claramente

13 Toharia (2000; págs. 93 y 94).

${ }^{14}$ Por ejemplo, en Toharia (2000; págs. 95-97). 
decreciente, a finales del siglo pasado venía a representar el $30 \%$ del total de parados.

Si es cierto que, según distintos estudios econométricos analizados en Toharia $(1996 b)^{15}$, la probabilidad de salida del paro es menor (pero no nula) para las personas que buscan empleo únicamente inscribiéndose en las oficinas públicas de empleo que para los que mencionan, además, otros métodos, ello no puede tener la incidencia suficiente como cuestionar la cifra de personas paradas estimadas por la EPA de forma significativa.

En cualquier caso, en 2002 el INE siguiendo lo establecido en el Reglamento 1897/2000, de la Comisión Europea, introdujo un cambio muy relevante en la definición del paro de la EPA (convirtiéndose en el primer país de la UE que adaptó su definición de paro al citado Reglamento), consistente en excluir de esa situación a las personas que, declarando que el único método de búsqueda es la inscripción en una oficina pública de empleo, no ha tenido contacto con la oficina en las últimas cuatro semanas con la finalidad estricta de encontrar trabajo, excluyendo de esa consideración a los que contacten con la oficina exclusivamente para la renovación de la demanda o para cuestiones relacionadas con los cursos de formación ocupacional o para el empleo.

En un artículo que Luis Toharia escribe en colaboración con Luis Garrido y publicado en 2004 en Labour Economics" ${ }^{16}$, titulado "¿Que hace falta para ser (contado como) parado? El caso de España"; se investiga, precisamente, siguiendo el ejemplo español, hasta qué punto los cambios en la definición de desempleo introducidos en la nueva reglamentación comunitaria tienen sentido desde el punto de vista del mercado de trabajo. En el artículo se muestra que el colectivo de personas excluidas del paro, que forma parte del subconjunto del concepto de paro utilizado hasta entonces, supone una tasa de supresión del paro, en torno al 15\% en 2003 , pero, además, esa tasa es muy inestable en los diferentes trimestres considerados, en contra de lo que podría esperarse a priori, de que la tasa de supresión fuera relativamente constante.

Ahora bien, si este colectivo excluido, al que Luis Garrido y Luis Toharia califican de "buscadores pasivos de empleo", tiene, en general, una probabilidad de paso de no trabajar a encontrar empleo inferior a la de los que se pueden calificar de "buscadores activos de empleo", que bien

15 Luis Toharia en este artículo publicado en Ekonomiaz en 1996 resume distintos estudios econométricos realizados sobre la probabilidad de salida de empleo según distintas características, una de ellas es la de la utilización de distintos métodos de búsqueda de empleo.

${ }^{16}$ Garrido y Toharia (2004). Este artículo, traducido, se incluye en el Libro de Homenaje a Luis Toharia publicado en 2012 por el Ministerio de Empleo y Seguridad Social (Jimeno y Toharia, 2012). 
utilizan otros métodos distintos a la inscripción en una oficina pública de empleo o bien utilizando ese método utilizan también otros distintos, la probabilidad de encontrar empleo de ese colectivo de "buscadores pasivos de empleo" es muy superior al de las personas entrevistadas no buscadoras de empleo por ningún procedimiento, tanto que estén dispuestos a trabajar como que no estén dispuestos a trabajar, que siempre se ha considerado en la EPA como un grupo de personas inactivas. Ante esta situación la conclusión de la exclusión del colectivo de "buscadores pasivos de empleo", que los dos autores consideran como una solución política y no técnica, es que esta dicotomización del colectivo de los que no trabajan puede, al considerar de igual manera a colectivos inscritos en las oficinas de empleo que a los que han abandonado toda búsqueda de empleo, desdibujar y subestimar el preocupante problema del desempleo en Europa, en lugar de enriquecer el conocimiento de la compleja realidad de la relación de las personas con la actividad económica.

\section{El paro en las fuentes administrativas}

Como el debate sobre la calidad de los datos de paro de la EPA siempre estuvo ligado en España a si los datos de paro registrado eran una buena alternativa a ellos, la obra de Luis Toharia también trata extensivamente el problema de la calidad de dichos datos administrativos para medir el paro.

Luis Toharia fue uno de los pioneros y más insistentes críticos del uso por parte de distintos gobiernos de los datos de paro $^{17}$, y, especialmente, de lo que él denominó la "ominosa" tasa de paro"18. Ésta consistía en una tasa de paro que en el numerador contaba con la cifra de parados registrados del antiguo INEM y en el denominador la población activa según la EPA (es decir, la suma del total de ocupados y parados según la EPA en ambos casos). Evidentemente esa "tasa" no era tal, pues el numerador no estaba estrictamente contenido en el denominador. Pero, además, su uso significa que se está asumiendo de forma implícita que la diferencia entre el cómputo del paro registrado y del paro de la EPA corresponde a personas que estarían trabajando.

\footnotetext{
17 Toharia (1996 b; pág. 40, nota 4).

18 Aunque Luis Toharia nunca fue muy dado a participar en los medios de comunicación este asunto sí que le llevó a publicar artículos en la prensa. Por ejemplo, véase en El País del 1 de agosto de 2000 su artículo titulado "¿Una tasas de paro del 8,97? ¡Seamos serios!".
} 
A pesar de la incoherencia que suponía utilizar fuentes estadísticas no homogéneas ni comparables, esa tasa de paro registrado era ampliamente usada por las administraciones públicas (incluso se publicada oficialmente por parte del Ministerio de Trabajo) y por los medios de comunicación. Fruto de la constante influencia de Luis Toharia, dicha "tasa" de paro registrado acabó por desaparecer en 2004, cuando el Ministerio de Trabajo abandonó por fin dicha práctica. Este hecho se vio completado por parte del Ministerio de Trabajo en 2005 con la puesta en marcha del SISPE (cuya elaboración llevaba gestándose desde unos años antes) y supuso un gran avance en la gestión informática de los servicios públicos de empleo, en el cual Luis Toharia desempeñó un papel muy relevante, allanando el camino para que se aceptasen las nuevas cifras de paro registrado, claramente superiores a las que generaba en antiguo sistema ${ }^{19}$.

Como es obvio, aparte de diferencias en las cifras de algo tan sensible como el desempleo, el uso de la cifra de paro registrado por parte de las autoridades públicas era "interesante" porque era sustancialmente menor que la cifra estimada por la EPA. La investigación de Luis Toharia se centró en un primer momento (la década de los ochenta del siglo XX) en mostrar las diferencias metodológicas y en explicar las grandes dificultades para comparar de forma directa ambas fuentes (por ejemplo, en Toharia, 1988).

No obstante, en los primeros años de la década de los noventa, Luis Toharia pronto percibió que la brecha entre las cifras de paro registrado y de paro de la EPA se estaba abriendo sin que mediasen cambios metodológicos explícitos en el paro registrado y sin que los cambios realizados en la EPA pudiesen explicar esa brecha. Como explica en Toharia (2000) la introducción de filtros informáticos en el cálculo del paro registrado estaba detrás de la evolución de esa brecha debido sobre todo a un problema de una inadecuada actualización de las claves de situación de los demandantes de empleo ${ }^{20}$. Así, una vez que un demandante de empleo resultaba excluido del cómputo del paro registrado por pertenecer a

\footnotetext{
${ }^{19}$ El detalle técnico de la implantación del SISPE y su impacto sobre las cifras de paro registrado aparece en Toharia y Malo (2005).

20 Hasta donde hemos podido averiguar, la primera ocasión en que Luis Toharia presentó este análisis en un foro público fue en la ponencia "How can unemployment in Spain be so high?", presentada en las Primeras Jornadas de Economía Laboral, celebradas en Alcalá de Henares (Madrid), del 7 al 9 de junio de 1995. El argumento principal de dicha ponencia (con más y mejores datos) se convirtieron en el artículo Toharia (2000), publicado en el número inaugural de la Revista Gallega de Empleo. No obstante, ya incluyó el argumento de la importancia de las exclusiones de demandantes en alguna publicación previa, como Toharia (1996a).
} 
alguna de las categorías de las que se presumía una baja disponibilidad (incluidas en la Orden Ministerial de 1985 sobre la definición oficial de paro registrado) quedaba excluido para siempre del cómputo del paro registrado aunque su situación cambiase ${ }^{21}$. El propio sistema informático SILE (Sistema de Información Laboral y de Empleo) propiciaba esta inercia en la exclusión, ya que tan sólo podía corregirse con una actualización realizada ex profeso para un individuo, algo muy extraño debido a que desde finales de los años ochenta las exclusiones pasaron a realizarse de forma automatizada a través de cruces con ficheros informáticos externos al antiguo INEM. Cuando el proceso de automatización de los filtros terminó a mediados de los años noventa, la brecha entre paro registrado y paro de la EPA volvió a estabilizarse.

Los trabajos de Luis Toharia hicieron cobrar conciencia al resto de investigadores de este problema, pero sobre todo lo consiguió con la propia Administración Pública. En 2005, junto con la implantación del nuevo sistema de gestión informática SISPE en las oficinas públicas de empleo, se corrige el anterior problema de desactualización de las claves (y deja de excluirse a los extranjeros). Por tanto, a partir de dicho momento la brecha entre paro registrado y el paro de la EPA está relacionada con las diferencias metodológicas, básicas y muy profundas entre ambas fuentes.

Merece la pena destacar que estos trabajos de Luis Toharia no tuvieron como objetivo acabar con la estadística de paro registrado, sino dotarla de mayor calidad e intentar circunscribir su uso a aquellas situaciones en las que la EPA es incapaz de llegar por su carácter de encuesta muestral. Así, Luis Toharia siguió usando este tipo de datos administrativos para analizar la evolución del paro en territorios muy reducidos donde la EPA no puede tener representatividad estadística y también para análisis dinámicos para los cuales la EPA no proporciona un periodo de tiempo lo suficientemente largo $^{22}$. Al mismo tiempo, sus trabajos sobre paro registrado suelen proporcionar a los usuarios de datos administrativos un aviso para navegantes: los incentivos (o desincentivos) para figurar en el registro del cual se obtienen los datos administrativos puede afectar sensiblemente el conjunto de personas de las cuales

${ }^{21}$ A esto se añadía el problema de la exclusión de los extranjeros al calcular la cifra de paro registrado sin que la Orden Ministerial de 1985 estableciese dicha exclusión. Como es lógico al principio esta exclusión no creaba distorsiones apreciables, pero conforme fue creciendo el volumen de trabajadores extranjeros en España esta exclusión se volvió muy relevante. Este problema también se corrigió en 2005 con la implantación del SISPE.

22 Dado que los entrevistados en la EPA permanecen en la muestra durante seis trimestres consecutivos no es posible seguir la trayectoria laboral de los individuos más allá de un año y medio. 
poseemos información y afectar por tanto a los análisis y conclusiones que se elaboren con esas bases de datos administrativas.

\section{Conclusiones}

¿Es diferente la medición del empleo y del paro en España gracias a las aportaciones de Luis Toharia en los últimos veinticinco años? La respuesta es afirmativa con total claridad. Ha participado no sólo en los cambios metodológicos de la EPA y del paro registrado, sino que también ha participado en el largo debate sobre si la EPA mide bien el desempleo, aportando razones y argumentos sobre las bondades de la EPA cuando el debate social sólo parecía atender a "argumentos" indemostrables y espúreos (como "todo el mundo miente en las encuestas"). De hecho, sus análisis mostraron antes que muchos otros que los problemas de la EPA afectaban más a la medición del empleo (por infraestimación) que al desempleo. Por otro lado, más allá del simplismo de denostar y desechar los datos administrativos, su investigación mostró que, entendiendo sus límites, los datos administrativos son una fuente insustituible hoy por hoy en España para analizar diferentes cuestiones sobre empleo y paro. Finalmente, hay que agradecerle especialmente que colaborase en que desde mediados de 2005 la ominosa tasa de paro registrado ya no castigue nuestra inteligencia desde los medios de comunicación ni desde las notas de prensa de las administraciones públicas.

\section{Bibliografía}

Bentolila, S. y Toharia, L. (1991): Estudios de Economía del Trabajo en España III. El problema del paro. Colección Economía y Sociología del Trabajo. Ministerio de Trabajo y Seguridad Social. Madrid.

Fina, LL, y Toharia, L. (2001): El empleo en España: Situación y perspectivas. Colección Informes y Estudios. Ministerio de Trabajo y Asuntos Sociales. Madrid.

Garrido, L., Requena, M.; y Toharia, L. (2000): "La Encuesta de Población Activa desde la perspectiva de los hogares". Estadística Española, 42 (146), 115-152.

Garrido, L. y Toharia, L. (2004): "What does it take to be (counted as) unemployed? The case of Spain”. Labour Economics, 11(4), 507-523. Reproducida su traducción en Jimeno, J.F. y Pérez Infante, J.I. (editores) 
(2012), con el título "¿Qué hace falta para ser (contado como) parado? El caso de España”, 287-307.

Jimeno, J.F. y Pérez Infante, J.I. (editores) (2012): El mercado de trabajo en la obra de Luis Toharia, Colección Economía y Sociología del Trabajo. Ministerio de Empleo y Seguridad Social de Madrid.

Muro, J.; Raymond, J.L.; Toharia, L.; y Uriel, E: "La encuesta de población activa y la encuesta de condiciones de vida y de trabajo". Estadística Española, 30 (119), 379-405.

Pérez Infante, J.I. (2006): Las estadísticas del mercado de trabajo en España. Colección Informes y Estudios. Empleo. Ministerio de Trabajo y Asuntos Sociales. Madrid.

Toharia, L. (1988): "Los parados en España. Cómo los medimos, cuántos hay y cuántos habrá". Debats, 25, 59-66. Reproducido en Bentolila, S. y Toharia, L. (1991), 75-88.

Toharia, L. (1994): "Empleo y paro”. Capítulo 8 del V Informe FOESSA, 1.227-1.410.

Toharia, L. (1996a): "La evolución del empleo y el paro en España". Cuadernos de Investigación Económica, 108, 20-27. Reproducido en Jimeno, J.F. y Pérez Infante, J.I. (editores) (2012), 205-229.

Toharia, L. (1996b): "Empleo y paro en España: Evolución, situación y perspectivas". Ekonomiaz, 35, 36-67.

Toharia, L. (director) (1998): El mercado de trabajo en España, McGraw Hill. Madrid.

Toharia, L. (2000): "El paro en España: ¿puede ser tan alto?”. Revista Gallega de Empleo, 0, 75-104.

Toharia, L. (2001): "La evolución del empleo y el paro en España. Un intento de construir una serie homogénea", en Fina, Ll. y Toharia, L. (2001), 63-92.

Toharia, L. y Malo, M.A. (2005): "La influencia de la implantación del SISPE en el paro registrado", Servicio Público de Empleo Estatal (antiguo INEM). 